\title{
Comparison of two interventions of increased blood flow rate and high-flux filters on hemodialysis adequacy and complications; a quasi-experimental study
}

\author{
Hosien Shahdadi ${ }^{1}$, Abbas Balouchi ${ }^{2,3}$, Maryam Jahantigh Haghighi ${ }^{*}$ \\ ${ }^{1}$ Department of Nursing, Faculty of Nursing and Midwifery, Zabol University of Medical Sciences, Zabol, Iran \\ ${ }^{2}$ Department of Nursing, Student Research Committee, Nursing and Midwifery School, Zabol University of Medical Sciences, Zabol, Iran \\ ${ }^{3}$ Student Research Committee, School of Nursing and Midwifery, Iran University of Medical Sciences, Tehran, Iran
}

\section{A R T I C L E I N F O}

Article Type:

Original

\section{Article History:}

Received: 27 February 2017

Accepted: 2 June 2017

Published online: 25 June 2017

\section{Keywords:}

Blood flow rate

High-flux filter

Dialysis adequacy

Dialysis complications

\begin{abstract}
A B S T RAC T
Introduction: Various parameters such as increased blood flow and high flux filter increase dialysis adequacy. Each parameter is associated with specific complications.

Objective: The aim of this study was comparison of two interventions of increased blood flow rate and high-flux filter on hemodialysis adequacy and complications.

Patients and Methods: This was a single-group quasi-experimental before-and-after intervention study. Twenty-two patients undergoing dialysis three times a week in the last 6 months consented to participate in the study. The participants were selected using random sampling method. They were reevaluated prior to dialysis and every 30 minutes until the end of each hemodialysis session using dialysis complication checklist. Dialysis adequacy was measured at the end of the fourth session for each patient.

Results: The paired t test results showed a significant increase in dialysis adequacy in dialysis with increased blood flow and dialysis with high flux filter (high-flux hemodialysis) compared to routine dialysis $(P=0.01)$. A significant increase was found in incidence of muscular cramp in dialysis with increased blood flow compared to routine dialysis based on McNemar's statistical test $(P=0.02)$.

Conclusion: Dialysis adequacy improved in both increased blood flow intervention and highflux hemodialysis compared to routine dialysis. On the other hand, increased blood flow intervention was associated with less complications than high-flux hemodialysis. In addition, increased blood flow intervention was more efficient and safer than high-flux hemodialysis.
\end{abstract}

Implication for health policy/practice/research/medical education:

Dialysis should be a safe procedure with less complications. It should improve physical state of the patients. High dialysis adequacy should also be taken into account in dialysis procedure. Thereby, the present study recommended dialysis with increased blood flow rate with higher adequacy and less complications compared to routine dialysis despite insignificant difference in dialysis adequacy and complications between increased blood flow intervention and high-flux hemodialysis. Nevertheless, increased blood flow intervention increased dialysis adequacy. For this purpose, this intervention was preferred over high-flux hemodialysis. Please cite this paper as: Shahdadi H, Balouchi A, Jahantigh Haghighi M. Comparison of two interventions of increased blood flow rate and high-flux filters on hemodialysis adequacy and complications; a quasi-experimental study. J Renal Inj Prev. 2017;6(4):247-252. DOI: 10.15171/jrip.2017.47.

\section{Introduction}

There are 1.8 million patients with end-stage renal disease (ESRD) around the world that should be treated with renal replacement therapy including hemodialysis, peritoneal dialysis or kidney transplant (1). By the end of 2014, 27457 dialysis patients were found in Iran (2). Of these,
$94 \%$ were treated with hemodialysis. The prevalence of hemodialysis was 300-400 in every one million people in Sistan and Baluchistan province by the end of 2014 (3). Dialysis inadequacy is a major cause of mortality in hemodialysis patients, which can cause such complications as dialysis, poor nutrition, nausea, vomiting, anorexia, 
hypoalbuminemia, restless leg syndrome, insomnia, hypertension, pericarditis, electrolyte imbalances and headache $(4,5)$. High adequacy of dialysis can relieve these complications. Various factors including dialysis duration and time, increased dialysate flow rate, high-flux filter and increased blood flow affect dialysis adequacy (5). In patients who cannot tolerate longer than 4-hour dialysis session, longer dialysis sessions are costly. Increased dialysate flow rate does not greatly affect dialysis adequacy (6). Confounding results were in dialysis adequacy with increased blood flow and high-flux filter interventions. Some studies suggested that increased rate of blood flow in hemodialysis device increases dialysis adequacy without increasing duration and cost of dialysis (7). Different studies showed that high blood flow is a convenient tool to increase dialysis adequacy $(8,9)$. However, increasing blood flow can also decrease dialysis adequacy due to vascular access type, hypotension and muscular cramps. This also leads to intolerance of continuous dialysis (10). There are no certain and determinative results on dialysis adequacy using high-flux filter. Various studies suggested that high-flux filters decrease mortality rate and improve clinical outcomes $(11,12)$. Most clinical guidelines also emphasize high-flux hemodialysis rather than low-flux hemodialysis (13). On the other hand, some studies suggested that high-flux filters cannot be practically used at all dialysis sessions for all patients because these filters are not often economically affordable. The patients cannot also tolerate these filters for long periods (14). Munshi et al showed that high-flux dialysis increases the risk of hemodynamic instability and disequilibrium syndrome although high-flux filters accelerate urea clearance more than low-flux filters at a zero ultrafiltration rate and blood flow and low dialysate flow rate (15). Although recent studies suggest that patients cannot tolerate this type of filters, Kavyannejad et al showed no statistically significant difference of high-flux and low-flux filters regarding such complications like nausea, vomiting, hypotension, fever, chills, headache, muscular pain and cramps. They showed no significant of mentioned items in dialysis patients using high-flux versus low-flux filters. However, the patients are more comfortable with using high-flux filters and better tolerate these filters than low-flux filters (16). Different studies also emphasized use of these filters to increase dialysis adequacy $(16,17)$.

\section{Objectives}

The present study aimed to compare two interventions of increased blood flow and high-flux filters with routine dialysis regarding dialysis complications and adequacy and also to select the more suitable method according to following issues. (a) Various studies indicated dialysis inadequacy for more than half of patients in Iran (6). (b) Increased blood flow increases and high-flux filter can be effective in improving dialysis adequacy. (c) Previous studies have recommended none of the above interventions for increasing dialysis adequacy. It is not clear whether these methods are preferable despite complications or not.

\section{Patients and Methods}

\section{Participants}

This semi-experimental, single-group before-and-after intervention study conducted on 22 hemodialysis patients in dialysis center of Zabol University of Medical Sciences from March 2015 to February 2016 in southeast of Iran. Patients were selected using random sampling method. Sample size calculated by Cochrane formula and related literatures $(18,19)$ with confidence interval $95 \%$ was 22 participants.

\section{Inclusion criteria}

Inclusion criteria were ESRD undergoing dialysis three times a week (each session lasting for 4 hours), having history of hemodialysis for $\geq 6$, having fistula, dialysis tolerance and willingness to participate in the study.

\section{Exclusion criteria}

Exclusion criteria were; having history of cardiopulmonary diseases, ultrafiltration rate less than three liters per dialysis session, age $\geq 15$ (years).

\section{Procedure}

The aim and method of the study were explained to the participants. The study was conducted in three phases. Each phase covered four dialysis sessions. In the first phase, all patients underwent routine dialysis sessions with 250 blood flow rate, low-flux filter and $500 \mathrm{~mL} /$ min dialysate flow rate. In the second phase, the patients underwent four dialysis sessions with increased blood flow rate. Accordingly, initial blood flow rates $(250 \mathrm{~mL} /$ $\min$ ) increased by $15 \%$ and $20 \%$ for the patients less than and more than $65 \mathrm{~kg}$ while keeping all other parameters constant. In the third phase, all patients underwent four sessions of high-flux hemodialysis. It is necessary to mention that, all patients were monitored regarding exclusion criteria, namely anti-cramp medications, nausea and vomiting 4 hours prior to the study, 100/60 $\mathrm{mm} \mathrm{Hg}<$ blood pressure $<140 / 90 \mathrm{~mm} \mathrm{Hg}$ at the onset of hemodialysis, smoking one hour prior to onset of dialysis, nausea and vomiting and muscular cramps before each session and changes in diet during the study. All the patients with the above criteria were excluded from the study. All patients were directly monitored before and after every dialysis session for detection of any complication. The B. Braun hemodialysis machine was used for all patients in each hemodialysis session under conditions of $37^{\circ}$ dialysate temperature, soluble bicarbonate dialysate, dialysate constant concentration and $140 \mathrm{mEq} / \mathrm{L}$ sodium concentration. All other parameters were kept constant for each patient, namely hemodialysis shift, ultrafiltration rate, use of supplemental caffeinated beverages before and during hemodialysis, diet and use of antihypertensive medications before dialysis. Blood samples were taken from the patients at the beginning and the end of the last dialysis session at each phase to determine dialysis adequacy. One blood sample was taken from arteries before dialysis and after injecting dialysis needle. Another 
blood sample was taken after dialysis before disjoining the patients from hemodialysis device. First, blood flow rate in the device was reduced to $50 \mathrm{~mL} / \mathrm{min}$. Then, blood samples were taken from dialysis needle injection region 15 to 30 seconds after reducing blood flow rate. The samples were immediately transferred to the laboratory. Blood urea nitrogen (BUN) was assessed before and after the fourth session at every phase of the study by urea standard Kt/V (stdKt/V) and Daugirdas 2 formula to determine dialysis adequacy (20). Weight of the patients was also measured before and after dialysis.

\section{Checklist}

A survey included demographic characteristics, dialysis complications list (Hypotension, muscular cramp, headache, nausea, vomiting) and dialysis adequacy (BUN, $\mathrm{KT} / \mathrm{V})$ used for data recording.

\section{Ethical issues}

The research followed the tenets of the Declaration of Helsinki; informed consent was obtained, and the research was approved by ethics committee of Zabol University of Medical Sciences. In this study, the full description of the processes and the importance of the study were explained to the patients who had volunteered and were selected. All of the assessments were non-invasive.

\section{Statistical analysis}

Descriptive tests of the frequency, mean and standard deviation (SD) were used to describe sample demographics. The Kolmogorov-Smirnov test was used to evaluate data normality paired $t$ test and McNamara's tests were applied to interpret results. SPSS version 18.0 for Windows (SPSS Inc., Chicago, IL, USA) was used to analyze the data. Confidence interval of $95 \%$ and a significance level of less than 0.05 was considered significant.

\section{Results}

Mean age of the participants was $43 \pm 3.21$. The most participants were females (60\%) and married (59.1\%). Mean score of dialysis adequacy was $0.83 \pm 0.22$ in routine dialysis, and $1.19 \pm 0.45$ in increased blood flow intervention. No statistically significant difference was found between routine dialysis and increased blood flow intervention regarding dialysis adequacy based on paired $t$ test results $(P<0.001)$. Mean score of dialysis adequacy was $1.02 \pm 0.30$ in high-flux hemodialysis. A significant increase was observed in high-flux hemodialysis compared to routine dialysis based on paired $t$ test results $(P=0.006)$. The findings showed that Kt/V was not acceptable in none of the patients undergoing routine dialysis (above 1.2). However, Kt/V was above 1.2 in $40.9 \%$ of the patients and equal to 1.2 in $9.09 \%$ of the patients in increased blood flow intervention. In addition, $\mathrm{Kt} / \mathrm{V}$ was above 1.2 in $36.3 \%$ of the patients undergoing high-flux hemodialysis. No statistically significant difference was found in mean $\mathrm{Kt} / \mathrm{V}$ between increased blood flow intervention and high-flux hemodialysis based paired $t$ test results $(P>$ 0.05 ; Table 1).

The incidence of muscular cramps was $6.81 \%$ in routine dialysis and $27.27 \%$ in increased blood flow intervention. A statistically significant difference was found between routine dialysis and increased blood flow intervention in incidence of muscular cramps based on McNemar's statistical test $(P=0.001)$. The incidence of hypotension in increased blood flow intervention was $15.91 \%$. The incidences of headache, nausea and vomiting were also $21.59 \%, 1.14 \%$ and $0 \%$ respectively in this intervention. No statistically significant difference between routine dialysis and increased blood flow intervention regarding hypotension, headache, and nausea and also vomiting was found (McNemar's statistical test) $(P>0.05)$. The incidence of hypotension and muscular cramps was $6.81 \%$ in routine dialysis and $15.91 \%$ and $19.31 \%$ in high-flux hemodialysis. Therefore, no statistically significant difference between routine dialysis and highflux hemodialysis regarding incidence of hypotension $(P=0.008)$ and muscular cramps $(P=0.003)$ was detected (McNemar's statistical test). The incidence of headache in routine dialysis and in high-flux hemodialysis was $20.45 \%$ and $20.45 \%$, respectively. The incidences of nausea and vomiting were respectively as $6.81 \%$ and $1.13 \%$ in routine dialysis and $1.14 \%$ and $0 \%$ in high-flux hemodialysis. No statistically significant difference between routine dialysis and high-flux hemodialysis regarding incidence of nausea and vomiting was seen (McNemar's statistical test) $(P>0.05$; Table 2$)$.

No significant difference of increased blood flow intervention with high-flux hemodialysis regarding incidence of dialysis complications was seen too (McNemar's statistical test) $(P>0.05$; Table 2$)$.

\section{Discussion}

The result of our study showed, dialysis adequacy was less than international targets (9), which is consistent with the results of different studies $(6,8)$. The results of this

Table 1. Comparison of mean $\mathrm{Kt} / \mathrm{V}$ in routine dialysis, increased blood flow intervention and high-flux hemodialysis

\begin{tabular}{|c|c|c|c|c|c|}
\hline \multirow{2}{*}{ Variable } & \multicolumn{2}{|c|}{ Method } & \multirow{2}{*}{$t$} & \multirow{2}{*}{$d f$} & \multirow{2}{*}{$P$} \\
\hline & Routine & Increased blood flow & & & \\
\hline \multirow[t]{5}{*}{ Mean Kt/V } & $0.83 \pm 0.22$ & $1.19 \pm 0.45$ & 4.40 & 21 & 0.001 \\
\hline & Routine & High-flux filter & & & \\
\hline & $0.83 \pm 0.22$ & $1.02 \pm 0.30$ & 3.03 & 21 & 0.006 \\
\hline & Increased blood flow & High-flux filter & & & \\
\hline & $1.19 \pm 0.45$ & $1.02 \pm 0.30$ & 1.86 & 21 & 0.07 \\
\hline
\end{tabular}


Table 2. Comparison of incidence of hemodialysis complication in routine dialysis, increased blood flow intervention and high-flux hemodialysis

\begin{tabular}{|c|c|c|c|}
\hline \multirow{2}{*}{ Variable } & \multicolumn{2}{|c|}{ Method } & \multirow{2}{*}{$P$} \\
\hline & Routine & High-flux & \\
\hline Hypotension & $6.81 \%$ & $15.91 \%$ & 0.008 \\
\hline \multirow[t]{2}{*}{ Muscular cramp } & $6.8 \%$ & $19.31 \%$ & 0.003 \\
\hline & Routine & Increased blood flow & \\
\hline Hypotension & $6.81 \%$ & $11.36 \%$ & $>0.05$ \\
\hline \multirow[t]{2}{*}{ Muscular cramp } & $6.8 \%$ & $27.27 \%$ & 0.001 \\
\hline & $\begin{array}{l}\text { Increased } \\
\text { blood flow }\end{array}$ & High-flux filter & \\
\hline Hypotension & $11.36 \%$ & $15.91 \%$ & 0.05 \\
\hline Muscular cramp & $27.27 \%$ & $19.31 \%$ & \\
\hline Headache & $21.59 \%$ & $20.45 \%$ & \\
\hline Nausea & $1.14 \%$ & $1.14 \%$ & \\
\hline Vomiting & 0 & 0 & \\
\hline
\end{tabular}

study indicated, dialysis inadequacy in routine dialysis using conventional filters, blood flow and dialysate flow rate. It is essential to design a highly adequate safe dialysis procedure $(21,22)$.

The increase blood flow rate is associated with high dialysis adequacy. These results are consistent with the previous studies $(6,23)$. Thereby, $15 \%$ to $20 \%$ increase in blood flow led to an increase in urea clearance followed by an increase in dialysis adequacy due to increased diffusion mechanism. Urea clearance depends on blood flow rate in the filter. Urea clearance can be accelerated by increasing blood flow rate in a filter with certain KoA, which increases metabolite diffusion in blood flow to the highest rate. As a result, electrolytes and waste are removed from the blood flow faster that ultimately increases dialysis adequacy (24). Mean Kt/V was better in high-flux hemodialysis than routine dialysis. Thereby, dialysis adequacy has significantly increased in highflux dialysis compared to routine dialysis. Several studies reported an effective improvement in dialysis adequacy using high-flux filter $(25,26)$. Werner et al also reported more suitable clinical outcomes using high-flux filters than routine dialysis (27). High-flux filters contain larger pores for removal of toxins compared to conventional filters. Thus, high-flux filters remove small molecules such as urea and average-sized molecules such as beta- 2 microglobulin faster than conventional filters (28). Since BUN measurement reflects removal of all toxins, highflux filters quickly remove BUN and increase dialysis adequacy (29). No statistically significant difference was found between increased blood flow rate intervention and high-flux hemodialysis in mean Kt/V. Both interventions increased dialysis adequacy in an effective manner. To the best of our knowledge, no study has compared two interventions of increased blood flow and high-flux filters for increasing dialysis adequacy. Although Nafar et al did not simultaneously compare these methods for increasing dialysis adequacy, low blood flow and selection of an unsuitable filter were mentioned as major causes of dialysis inadequacy. Their results showed that dialysis adequacy was higher (above 1.4) in dialysis patients with high blood flow rates and lower physical weight. They also showed that a filter with $\mathrm{KoA}=700$ was administered for $79.3 \%$ of the patients to increase Kt/V by $1.4(30)$.

A significant difference between routine dialysis and increased blood flow intervention regarding muscular cramps was detected. No significant difference between routine dialysis and increased blood flow intervention was seen. Additionally a statistically significant difference of routine dialysis with high-flux hemodialysis in incidences of hypotension and muscular cramps was seen.

Different studies reported no statistically significant difference between routine dialysis and increased blood flow intervention in incidences of hypotension, headache, nausea and vomiting $(10,31)$. These results were consistent with the results of the present study. The results showed an improvement in dialysis adequacy in both interventions compared to routine dialysis. However, an increase in incidence of muscular cramps was reported in high-flux hemodialysis and an increase in incidences of hypotension and muscular crams were reported in increased blood flow intervention. Nevertheless, no significant difference in dialysis adequacy and complications between increased blood flow intervention and high-flux hemodialysis was existed.

\section{Conclusion}

Dialysis should be a safe procedure with less complications. It should improve physical state of the patients. High dialysis adequacy should also be taken into account in dialysis procedure. Thereby, the present study recommended dialysis with increased blood flow rate with higher adequacy and less complications compared to routine dialysis despite insignificant difference in dialysis adequacy and complications between increased blood flow intervention and high-flux hemodialysis. Nevertheless, increased blood flow intervention increased dialysis adequacy. For this purpose, this intervention was preferred over high-flux hemodialysis.

\section{Limitations of the study}

The convenience of participants and hard access to different filters were the limitations of our study.

\section{Acknowledgments}

We would like to thank all patients participating in this study and Zabol University of Medical Sciences for supporting our study.

\section{Authors' contribution}

Design and concept: HS and MJH. Data analysis: AB. Writing of the manuscript: MJH and HS. Data collection: $\mathrm{MJH}$ and $\mathrm{AB}$. Critical revision: $\mathrm{HS}, \mathrm{MJH}$ and $\mathrm{AB}$.

\section{Conflicts of interest}

The authors declare no conflict of interest.

Ethical considerations

Ethical issues (including plagiarism, data fabrication, 
double publication) have been completely observed by the authors.

\section{Funding/Support}

Zabol University of Medical Sciences supported the study (Thesis \# zbmu.1394.12.132).

\section{References}

1. Mills KT, Xu Y, Zhang W, Bundy JD, Chen C-S, Kelly TN, et al. A systematic analysis of worldwide population-based data on the global burden of chronic kidney disease in 2010. Kidney Int. 2015;88:950-7. doi: 10.1038/ki.2015.230

2. Mousavi SSB, Soleimani A, Mousavi MB. Epidemiology of end-stage renal disease in Iran: a review article. Saudi J Kidney Dis Transpl. 2014;25:697. doi: 10.4103/13192442.132242.

3. Pakfetrat $\mathrm{M}$, Dabbaghmanesh $\mathrm{MH}$, Karimi Z, Rasekhi A, Malekmakan L, Hossein Nikoo M. Prevalence of hypothyroidism and thyroid nodule in chronic hemodialysis Iranian patients. Hemodial Int. 2017;21:84-9. doi: 10.1111/ hdi. 12453.

4. Perl J, Dember LM, Bargman JM, Browne T, Charytan DM, Flythe JE, et al. The use of a multidimensional measure of dialysis adequacy-moving beyond small solute kinetics. Clin J Am Soc Nephrol 2017;12:839-847. doi: 10.2215/ CJN.08460816.

5. Zhang P, Wu H-M, Shen Q-Y, Liu R-Y, Qi X-M. Associations of pulmonary function with serum biomarkers and dialysis adequacy in patients undergoing peritoneal dialysis. J Clin Exp Nephrol. 2016;20:951-9. doi: 10.1007/s10157-0161244-1.

6. Shahdadi H, Balouchi A, Sepehri Z, Rafiemanesh H, Magbri A, Keikhaie F, et al. Factors affecting hemodialysis adequacy in cohort of Iranian patient with end stage renal disease. Glob J Health Sci. 2016;8:50. doi: 10.5539/gjhs. v8n8p50.

7. Richtrova P, Mares J, Kielberger L, Trefil L, Eiselt J, Reischig T. Citrate-buffered dialysis solution (citrasate) allows avoidance of anticoagulation during intermittent hemodiafiltration-at the cost of decreased performance and systemic biocompatibility. Artif Organs. 2016. doi: 10.1111/aor.12851.

8. Malekmakan L, Malekmakan A, Sayadi M, Pakfetrat M, Sepaskhah M, Roozbeh J. Association of high-sensitive C-reactive protein and dialysis adequacy with uremic pruritus. Saudi J Kidney Dis Transpl. 2015;26:890. doi: 10.4103/1319-2442.164565.

9. Slinin Y, Greer N, Ishani A, MacDonald R, Olson C, Rutks $\mathrm{I}$, et al. Timing of dialysis initiation, duration and frequency of hemodialysis sessions, and membrane flux: a systematic review for a KDOQI clinical practice guideline. Am J Kidney Dis. 2015;66:823-36. doi: 10.1053/j.ajkd.2014.11.031.

10. Salehi A, Shahgholian N, Mortazavi M. Investigation of the effects of stepwise sodium and ultrafiltration profile on dialysis adequacy. J Crit Care Nurs. 2016 ;9:e5105. doi: 10.17795/ccn-5105

11. Ok E, Asci G, Toz H, Ok ES, Kircelli F, Yilmaz M, et al. Mortality and cardiovascular events in online haemodiafiltration (OL-HDF) compared with high-flux dialysis: results from the Turkish OL-HDF Study. Nephrol Dial Transplant. 2013;28:192-202. doi: 10.1093/ndt/gfs407. 12. Kim HW, Kim S-H, Kim YO, Jin DC, Song HC, Choi
EJ, et al. Comparison of the impact of high-flux dialysis on mortality in hemodialysis patients with and without residual renal function. PloS One. 2014;9:e97184. doi: 10.1371/journal.pone.0097184.

13. Abecassis M, Bartlett ST, Collins AJ, Davis CL, Delmonico FL, Friedewald JJ, et al. Kidney transplantation as primary therapy for end-stage renal disease: a National Kidney Foundation/Kidney Disease Outcomes Quality Initiative $\left(\mathrm{NKF} / \mathrm{KDOQI}^{\mathrm{TM}}\right)$ conference. Clin J Am Soc Nephrol. 2008;3:471-80. doi: 10.2215/cjn.05021107.

14. Mostovaya IM, Blankestijn PJ, Bots ML, Covic A, Davenport A, Grooteman MP, et al. Clinical evidence on hemodiafiltration: a systematic review and a meta-analysis. Semin Dial. 2014;27:119-27.

15. Munshi R, Ahmad S. Comparison of urea clearance in lowefficiency low-flux vs. high-efficiency high-flux dialyzer membrane with reduced blood and dialysate flow: an in vitro analysis. Hemodial Int. 2014;18:172-4. doi: 10.1111/ hdi. 12054.

16. Kavyannejad R, Oshvandi K, Borzuo R, Gholyaf M. A comparison of the effects of two low- and high-flux dialyzers on the patient's comfort and hemodialysis complications: a randomized clinical trial. Qom Univ Med Sci J. 2015;9:11-9.

17. Li Y, Wang Y, Lv J, Wang M. Clinical outcomes for maintenance hemodialysis patients using a highflux (FX60) dialyzer. Ren Fail. 2013;35:1240-5. doi: $10.3109 / 0886022 x .2013 .823872$.

18. Borzou S, Gholyaf M, Zandiha M, Amini R, Goodarzi M, Torkaman B. The effect of increasing blood flow rate on dialysis adequacy in hemodialysis patients. Saudi J Kidney Dis Transpl. 2009;20:639.

19. Mareels G, Kaminsky R, Eloot S, VerdonckPR. Particle image velocimetry-validated, computational fluid dynamicsbased design to reduce shear stress and residence time in central venous hemodialysis catheters. Asaio Journal. 2007;53:438-46. doi: 10.1097/mat.0b013e3180683b7c.

20. Daugirdas JT. Second generation logarithmic estimates of single-pool variable volume Kt/V: an analysis of error. Clin J Am Soc Nephrol. 1993;4:1205-13.

21. Li W, Hongyun P, Bin Z, Shenglang Z, Jiuhong L, Huayun $Z$, et al. Effect of different puncture distances in internal arteriovenous fistula on dialysis adequacy in maintenance hemodialysis patient. Mod Clin Nurs. 2015;5:004.

22. Robinson BM, Akizawa T, Jager KJ, Kerr PG, Saran R, Pisoni RL. Factors affecting outcomes in patients reaching end-stage kidney disease worldwide: differences in access to renal replacement therapy, modality use, and haemodialysis practices. Lancet. 2016;388:294-306. doi: 10.1016/s01406736(16)30448-2.

23. Youssouf S, Nache A, Shnathappa A, Green P, Middleton RJ, Shurrab AE, et al. Long term outcomes over 2 years following a dialysis adequacy quality improvement initiative. Nephrol Dial Transplant. 2015;30:iii312. doi: 10.1093/ndt/gfv183.25.

24. Mandolfo S, Borlandelli S, Ravani P, Imbasciati E. How to improve dialysis adequacy in patients with vascular access problems. J Vasc Access. 2005;7:53-9.

25. Oshvandi K, Kavyannejad R, Borzuo SR, Gholyaf M. Highflux and low-flux membranes: efficacy in hemodialysis. Nurs Midwifery Stud. 2014;3:e21764. doi: https://doi. org/10.17795/nmsjournal21764

26. Meert N, Eloot S, Schepers E, Lemke H-D, Dhondt A, Glorieux G, et al. Comparison of removal capacity of two consecutive generations of high-flux dialysers during 
different treatment modalities. Nephrol Dial Transplant. 2011;26:2624-30. doi: 10.1093/ndt/gfq803.

27. Bosch W, Buck R, Gambro S. The Next Generation in HighFlux Dialyzers. Denver: Lakewood CO; 2009.

28. Kim HW, Kim S-H, Kim YO, Jin DC, Song HC, Choi EJ, et al. The impact of high-flux dialysis on mortality rates in incident and prevalent hemodialysis patients. Korean J Intern Med. 2014;29:774. doi: 10.3904/kjim.2014.29.6.774.

29. Dhondt A, Vanholder R, Van Biesen W, Lameire N. The removal of uremic toxins. Kidney Int. 2000;58:S47-S59. doi: 10.1046/j.1523-1755.2000.07606.x.

30. Nafar M, Samavat S, Khoshdel A, Abedi BA. Dialysis adequacy, dialyzer clearance, and strategies to achieve target: a nationwide multicenter study. Nephrourol Mon. 2016;9:42769. doi: 10.5812/numonthly.42769.

31. Trivedi HS, Kukla A, Prowant B, Lim HJ. A study of the extracorporeal rate of blood flow and blood pressure during hemodialysis. Hemodial Int. 2007;11:424-9. doi: 10.1111/j.1542-4758.2007.00212.x.

Copyright $\odot 2017$ The Author(s); Published by Nickan Research Institute. This is an open-access article distributed under the terms of the Creative Commons Attribution License (http://creativecommons.org/licenses/by/4.0), which permits unrestricted use, distribution, and reproduction in any medium, provided the original work is properly cited. 